\title{
QoS in UMTS Network and Improvement Voice over IP Performance
}

\author{
${ }^{1}$ Lamia Bakri Abd elhaleem Derar, ${ }^{2}$ Amin Babiker Abd elnabi Mustafa \\ ${ }^{I}$ Department of Communications, Faculty of Engineering, Alneelain University, Khartoum, Sudan \\ ${ }^{2}$ Department of Communications, Faculty of Engineering ,Alneelain University, Khartoum, Sudan
}

\begin{abstract}
This study adopted a simulation based network performance analysis to investigate the effects of the application of different voice encoder schemes on QoS of VoIP system, deployed with UMTS network Through different network simulation experiments using realistic network scenarios in OPNET environment, the results indicated that the choice of suitable voice encoder scheme with a small number of voice frame size per packet have a significant impact over VoIP traffic performance when deployed with UMTS access technology the VoIP over UMTS network model has been developed, where a VoIP server development is connected in the UMTS model the QoS factors will be controlled and managed to ensure good quality in VoIP call, the design of layered coding and multiple description coding is employed to address the bandwidth fluctuations and packet loss problems in the wireless network and to further enhance the error resilience, this research provided an in depth network performance comparative analysis of VoIP over UMTS using performance parameters which indicate QoS such as end to end packet loss, throughput, end to end delay, uplink traffic sent, uplink traffic received voice, downlink traffic sent, downlink traffic received, voice packet delay, average in voice packet end to end delay by using the GSM ,G729A Codec's in frames (20, $30 \mathrm{~ms})$. The obtained simulation experiment results indicated that choice of suitable codec scheme can affect the QoS of VoIP traffic over UMTS network.
\end{abstract}

Keywords: VOIP, OPNET, CODEC, UMTS, QOS, Delay, Throughput

\section{Introduction}

With the evolution of mobile networks and popularity of smart phones, more and more applications having Quality of Service requirements are coming up; mobile data services are penetrating mobile markets rapidly. The mobile industry relies heavily on data service to replace the traditional voice services with the evolution of the wireless technology and market. A reliable packet service network is critical to the mobile operators to maintain their core competence in data service market. Furthermore, mobile operators need to develop effective operational models to manage the varying mix of voice, data and video traffic on a single network. Application of statistical models could prove to be an effective approach each application requires different level of QoS according to the service. QoS is essential to maintain and provide better service for the users. Some applications are delay sensitive and some are not. Based on the sensitivity, the applications in UMTS network are categorized into different classes. The UMTS QoS requirements for some applications are mentioned in table 1 [1].UMTS (Universal Mobile Telecommunications Systems) and third generation (3G) telecommunication systems offer a wide range of Services to the users. One of the biggest challenges with the fast growth of multimedia applications over Internet is to maintain Quality of Service (QoS), meaning that the service through Internet should be guaranteed. Different methods are suggested to maintain QoS, even though it is not always possible to guarantee the quality of all requirements. Basically, the QoS requirements are translated into some specific variables that define the performance experienced by users. Thus, different QoS parameters are assigned to each user depending on the application data it carries, enabling therefore differentiation among them. To this purpose, different classes of QoS services have been defined by means of QoS Class Identifiers (QCIs), which are scalar values used as a reference for driving specific packet forwarding behaviors [2]. Each QCI is characterized by a resource type Guaranteed Bit Rate (GBR) or non GBR), a priority level, the maximum permitted packet delay as well as the acceptable packet loss rate. Since most traffic flows in the downlink (DL) side of the communication, this investigation is made to improve the performance on this link.

\subsection{Problem statement}

In Universal Mobile Telephone System (UMTS) there is some important parameters affect the system performance which leads the system to suffer degradation in quality of service (QoS). In this paper will focus on the study and the performance measurement for parameters affect on QoS specifications. Furthermore, In order to manage the resources and to handle the traffic properly in the network, to improve the overall network quality as experienced by the mobile subscribers and to ensure that the network resources are efficiently utilized. This includes performance measurements, analysis of measurement results and updates of the network configuration 
and parameters. QoS implementation is necessary to provide better service as well as to fulfill the users expectations, mobile operators need to develop effective operational models to manage the varying mix of voice, data on a single network. Application of simulation models could prove to be an effective approach.

\section{2 objectives}

The aim of this thesis work is to improving the QoS in UMTS (3G) network to be capable of satisfying the increasing data traffic and performance, evaluate the existing QoS performance of voice call in the UMTS network based on some important performance metrics such as packet loss, delay, queuing delay throughput, uplink and downlink traffic and average end to end delay in voice packet.

\subsection{Research Methodology}

We have used OPNET Modeler, in our simulation; we have designed different scenarios for voice conference and measure the performance of some parameters such as throughput, jitter, packet loss, queuing delay and average end to end delay in voice packet according to the Codec's. G729A and GSM Codec, uplink and downlink traffic, as well as meet the goal to analysis the quality voice in $3 \mathrm{G} / \mathrm{UMTS}$ wireless networks. Then we will evaluate the results improving the QoS to support in $3 \mathrm{G}$ network for successful transmission.

\subsection{Related Work}

There has been quite a lot of research done in the past, involving the quality of VoIP in $3 \mathrm{G}$ system and evaluated the performance analysis and QoS. Several techniques that are believed to bring improvements in VoIP quality have been proposed and techniques of how to control call and data congestion due to QoS factors. To initiate a VoIP call, at least, signaling protocols, that include, Session Initiation Protocol (SIP), H.323, H.248 (MEGACO) and MGCP [3] are required. SIP is defined in [4, 5]. In VoIP technology, a codec is essential for encoding and decoding speech. There are many types of codec's that can be used for this function.

[6] Proposed packet loss reduction to VoIP by means of AMR codec speech, whereby AMR codec maintains the toll quality of speech signals. According to [7], an AMR codec is a compulsory codec for conversational speech services within $3 \mathrm{G}$ systems. AMR codec consists of eight bit-rates which range from $4.5 \mathrm{kbps}$ to 12.2 kbps and it is able to switch its bit-rate every $20 \mathrm{~ms}$ of speech frame depending on channel and network conditions [8].

[7] Proposed an end-to-end quality of service analysis in VoIP over 3G networks, whereby they checked if jitter in $3 \mathrm{G}$ networks has a negative effect on the end-user voice quality. According to [1], an E-model technique evaluates the quality of VoIP in wireless networks. This E-model technique accepts a wide range of telephone damages into consideration, like damages due to low bit rate coding, one-way delay, echo and noise [9].

In [10] QoS in VoIP over 3G network and Pricing Strategy, guaranteeing end-to-end quality to a VoIP call over UMTS network is proposed, whereby the VoIP application parameters (voice codec, packet size and de-jittering delay), and UMTS air interface parameters (coding rate and interleaving span) are used. To ensure the quality of the VoIP call, the delay and the loss of voice packet over the air interface need a thorough control mechanism. A methodology to set VoIP application parameters and UMTS . Fezeka J. Mkhetshana, Karim Djouani and Guillaume Noel French South African Institute of Technology (F'SATI) Department of Electrical Engineering Tshwane University of Technology, P. O. Box 680, Pretoria 0001 Tel: +27 12 3824809, Fax: +27 123825294 Email: fezekam6@gmail.com, \{djouanik, NoelG\}@tut.ac.za air interface parameters, so that the quality of VoIP calls within a UMTS party is ensured, was developed .

In [11] Failure to future network upgrades can be due to non-profit to internet providers. Therefore, this may cause poor internet services that lead dissatisfaction to end-users. Pricing strategy is another tool used in realtime application to ensure QoS. The dynamic pricing that maximizes revenue while satisfying blocking rate target has been developed.

In [12], whereby a calculus of variations and Lagran gian mechanism to solve the carried load problem is utilized. This carried load may be the result of packet delay on the network.

In [13], a packet-marking based pricing scheme is proposed. This scheme is for networks with multiple service providers.

\section{VoIP and Codec's And Protocols}

\section{A. VoIP}

The demand for mobile and broadband services is rising day by day. The last decade has seen the everincreasing VoIP users with the demand of reliable and quality services. VoIP is an emerging technology for voice communication used these days. The services are not only being used for long distance calls but also for 
the short distant communications. The devices like IP phones and the VoIP enabled desktop systems are cost effective and also provide some new features to the users. Keeping in mind the demand of the users, the operators are forced to improve the quality of communication. This can be achieved by increasing the bandwidth and making the IP backhaul that fulfills the demand of the users at lower cost providing better QoS[14].

\section{B. VoIP Codec's}

Codec is a coder/decoder which converts the audio signal to digitized version for transmission over the medium and then back into the original uncompressed version on the receiver side. This concept is the base of VoIP services. There are a number of codec used for VoIP communication each having its own bandwidth and characteristics. The codec's which are used in this research work are The Codec's G729A and GSM. Codec uses different algorithms to compress and decompress the voice stream and each CODEC will contribute a processing delay to the overall end-to-end delay [14]. The popular Codec's are used in the Table 2 below.

\section{VoIP Protocols}

In a typical VoIP system, the voice stream is digitalized into voice frames. The voice frames are encapsulated and transmitted in RTP packets which are managed using RTCP which provides stream control and statistical information [15]. Signaling protocols have been introduced to provide overall management of VoIP calls. In the recent years, the Session Initiation Protocol (SIP) [16] has become very popular and is playing a major role in advancing VoIP.

\section{NETWORK MODELS:}

The tool used for simulations is OPNET Modeler as it provides the results very closer to the real time environment. The models were created by selecting the nodes and links from the object palette such that to reduce the losses and impairments effect

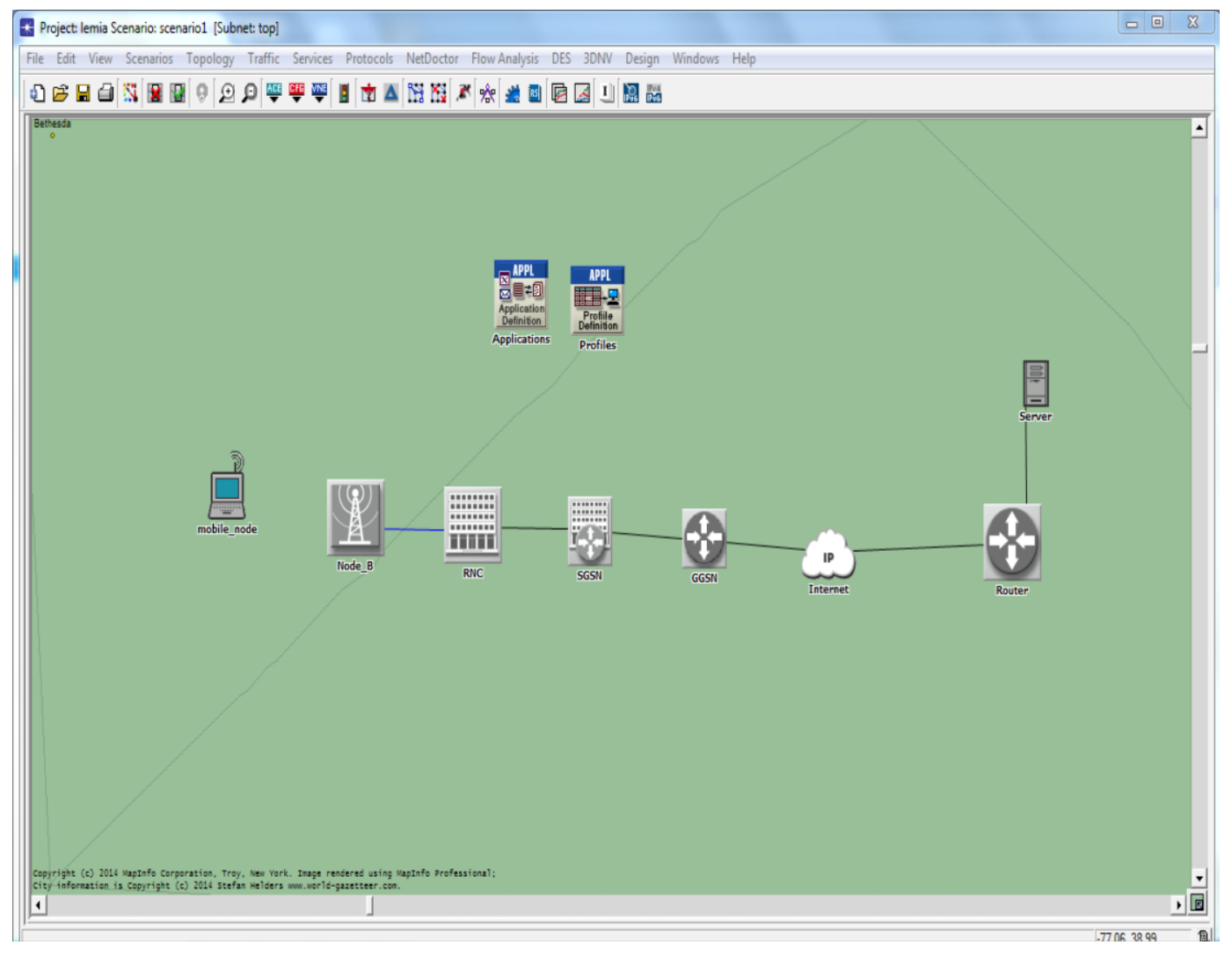

Fig 1: Simulation Environment

Network which is connected to the Internet via the GGSN. A workstation and a SIP server are connected to the Internet using a router. The SIP Server is used as a signaling server to establish VoIP calls between the UE and the workstation, conversational class has been used. To evaluate the performance of the QoS, we import IP QoS attribute to implement QoS in the network. Under the QoS, different types of queuing mechanisms are available. For our simulation, we test queuing scheme and as a result, we use this queuing mechanism in our simulation to 
obtain better performance, we used Differentiated Services Code Point (DSCP) for the different types of applications such as voice DSCP is used to mark the packets for classifying and forward these packets on the basis of Per Hop Behavior (PHB) that are associated with different traffic classes. PHB supports two types of forwarding scheme which are Expedited Forwarding (EF) and Assured Forwarding (AF); these are called Differentiated Service Code Point (DSCP). The scenario with QoS is depicted in figure [1].We design simulation model to evaluate the performance of network and QoS for voice transmission in UMTS network.

\section{Simulation Setup:}

In carrying out the network performance evaluation of voice over IP using UMTS network, we designed and simulated close to real life network scenarios to investigate the critical parameter that affect of overall performance in UMTS network, QoS of VoIP real time in a UMTS network using OPNET modeler network simulator. The simulation only considered VoIP supported services and applications. We used a server backbone with one voice server. In this simulation setup, we performed the following experiments:

Experiment 1: here we used scenario 1 simulation to study the performance and QoS parameters over UMTS network which leads the system to suffer degradation in quality of service (QoS) used in scenariol

Experiment 2: here we used scenario 2 simulation to study the effect of different CODEC on VoIP services over UMTS networks. The encoder schemes used for the investigation include G.729 codec, GSM codec with voice frame size used per packet set to $(20,30 \mathrm{~ms})$ and simulations have been done using the hybrid network. The G.729 and GSM frame CODEC with a $20 \mathrm{~ms}$ frame size has been configured and used with a varied number of voice call frames in each VoIP packet.

\section{Simulation Result}

This section contains the simulation result for scenario that is described below.

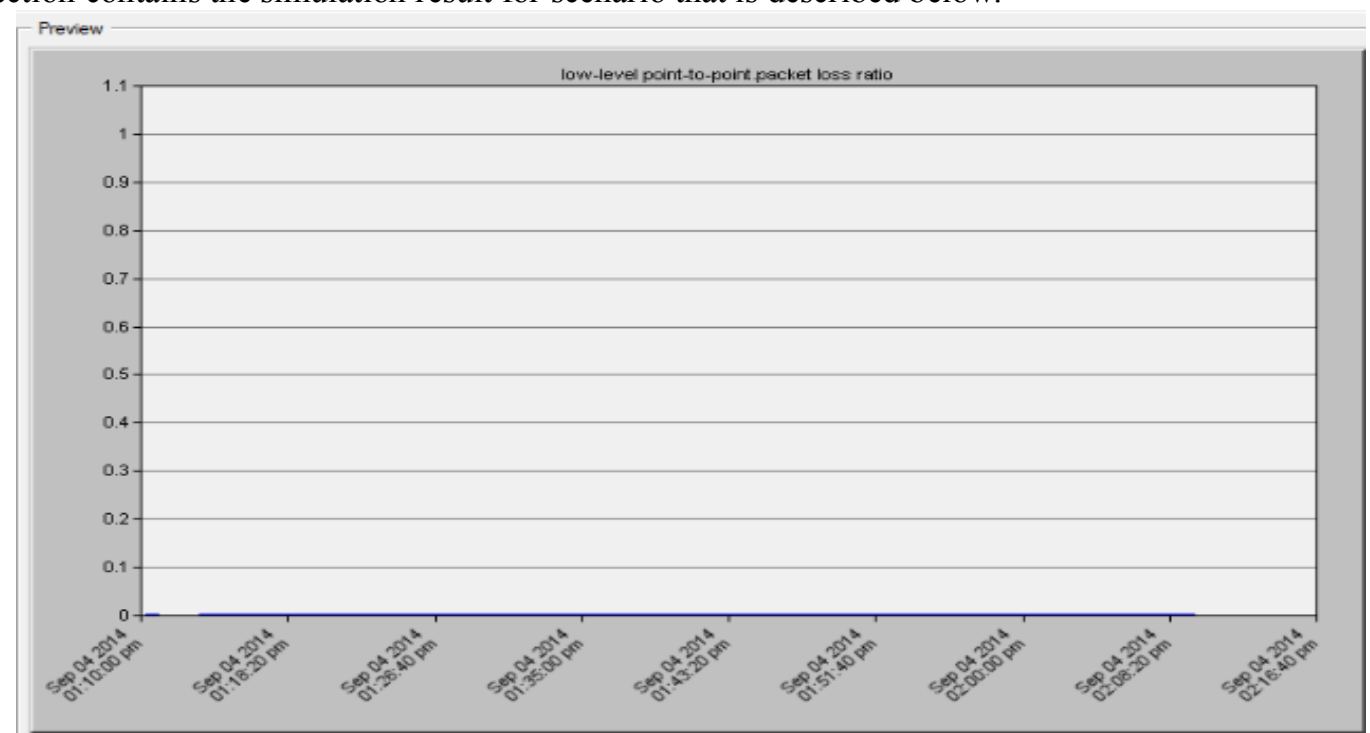

Fig 2: End to end packet loss 


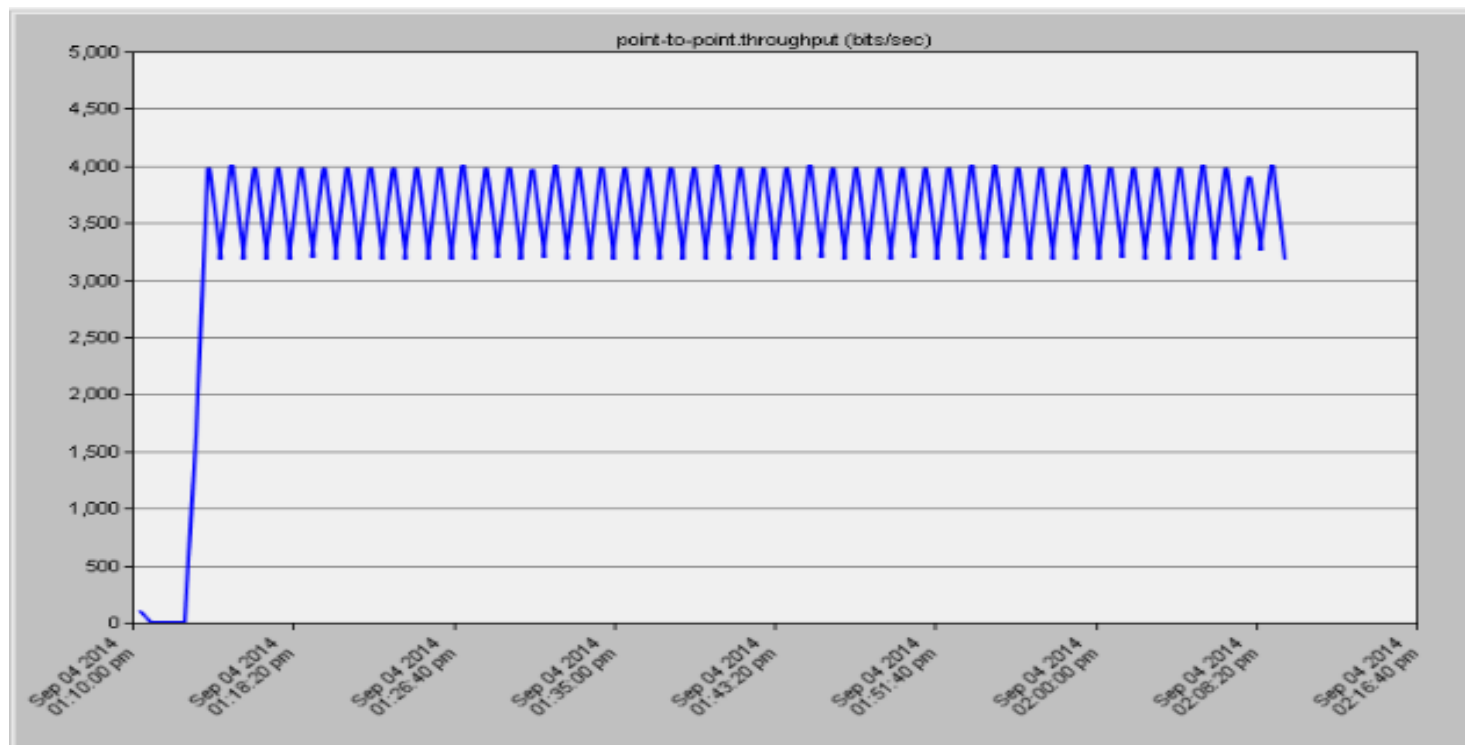

Fig 3: Throughput

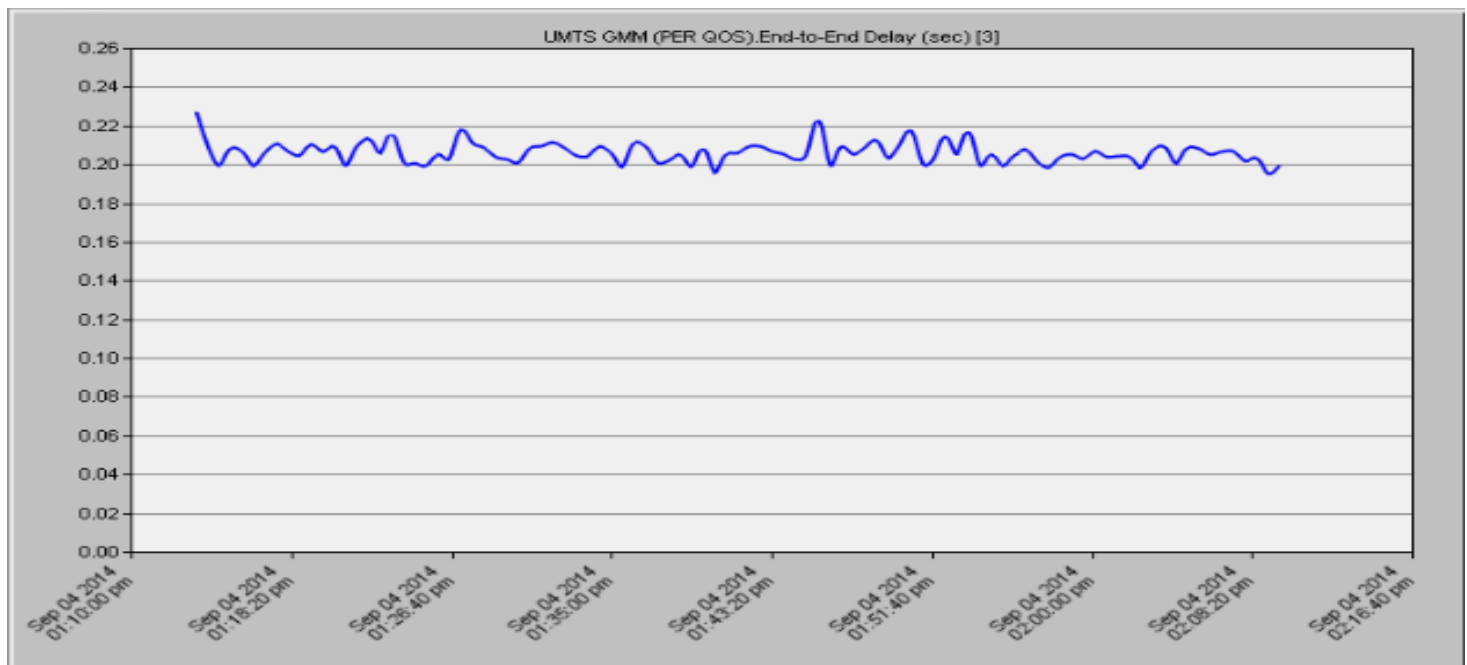

Fig 4: End to End Delay

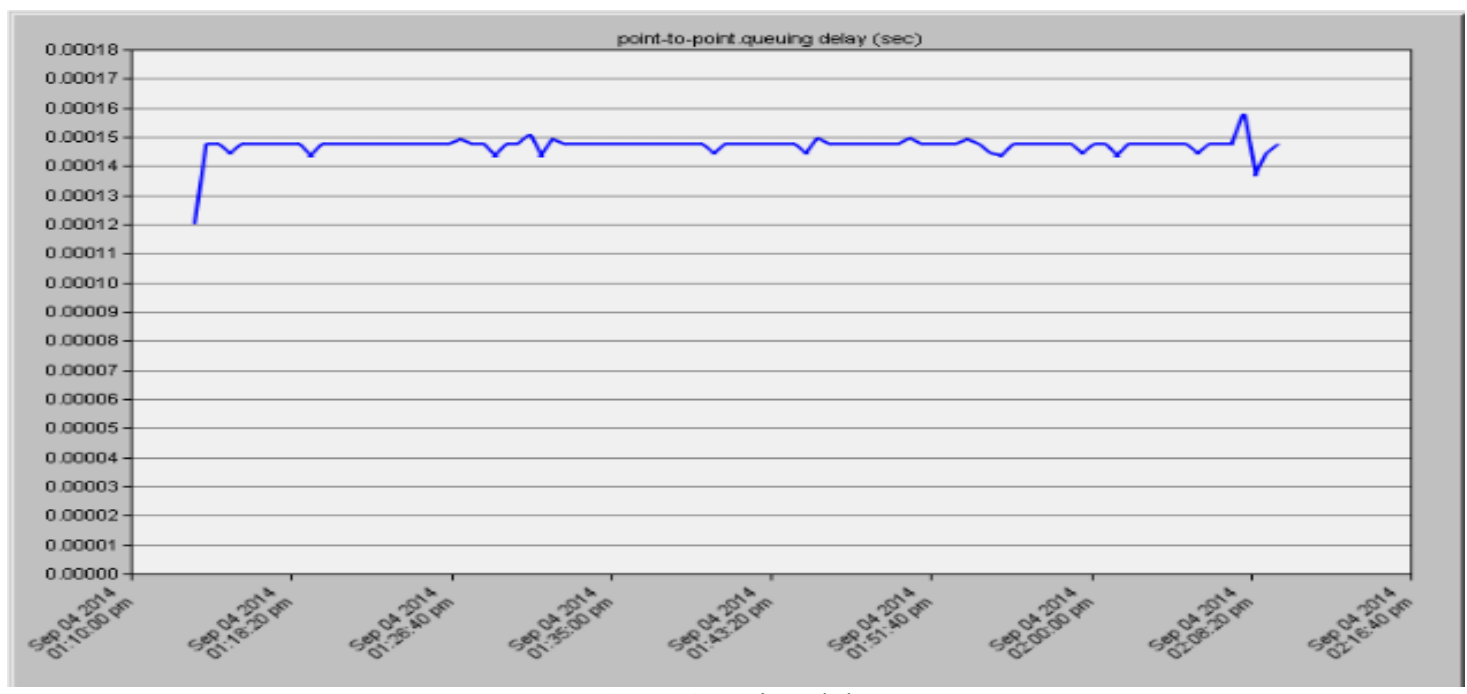

Fig 5: Queuing delay 


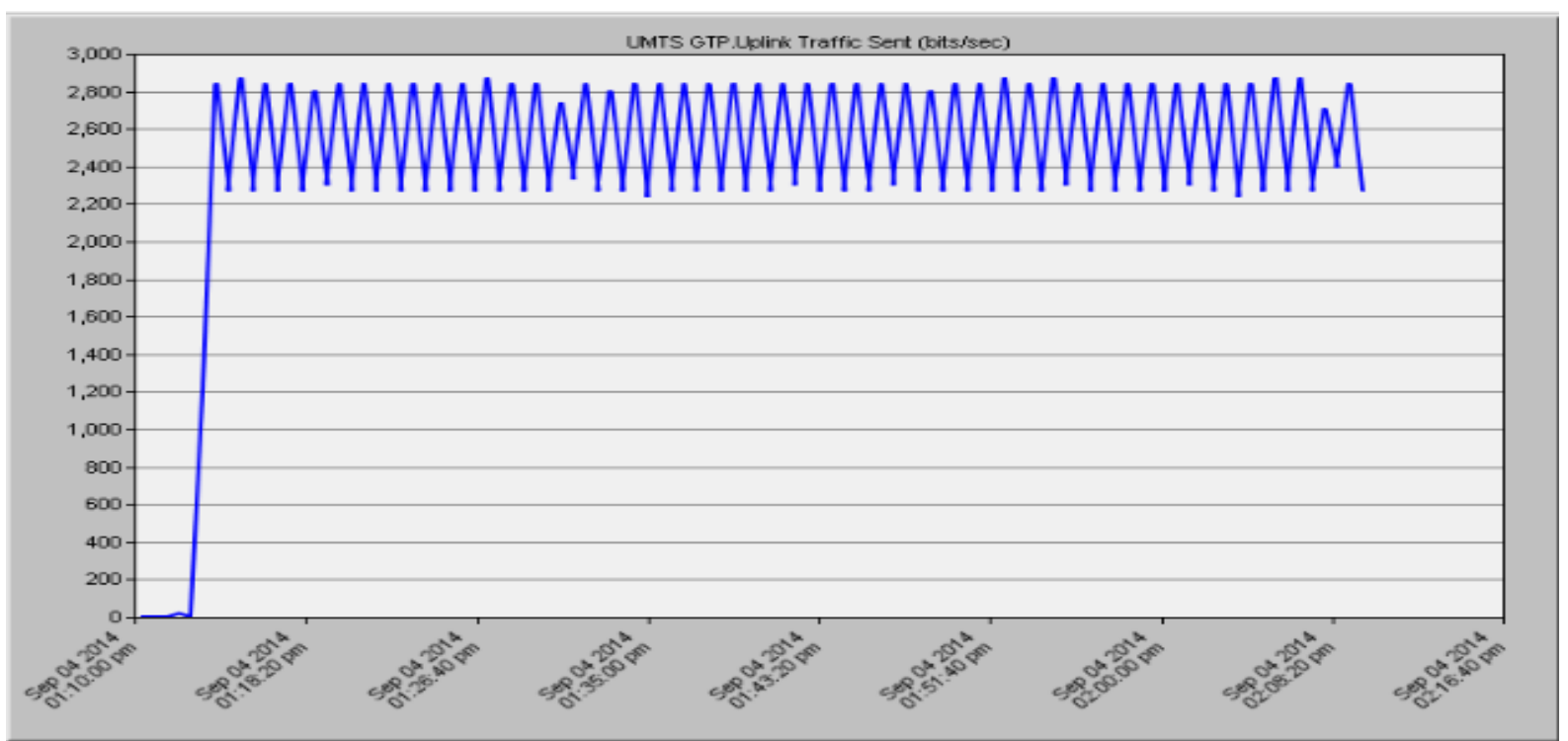

Fig 6: Uplink traffic sent

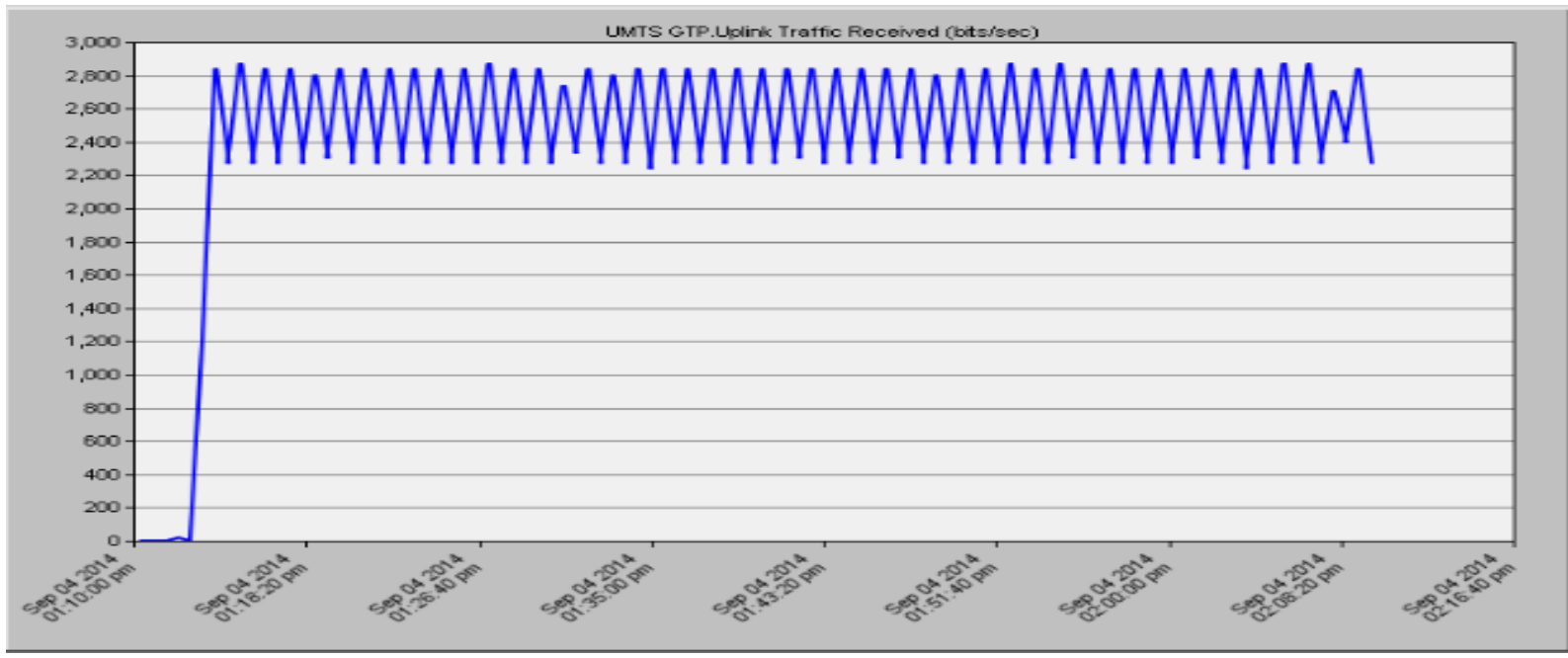

Fig 7: Uplink traffic received

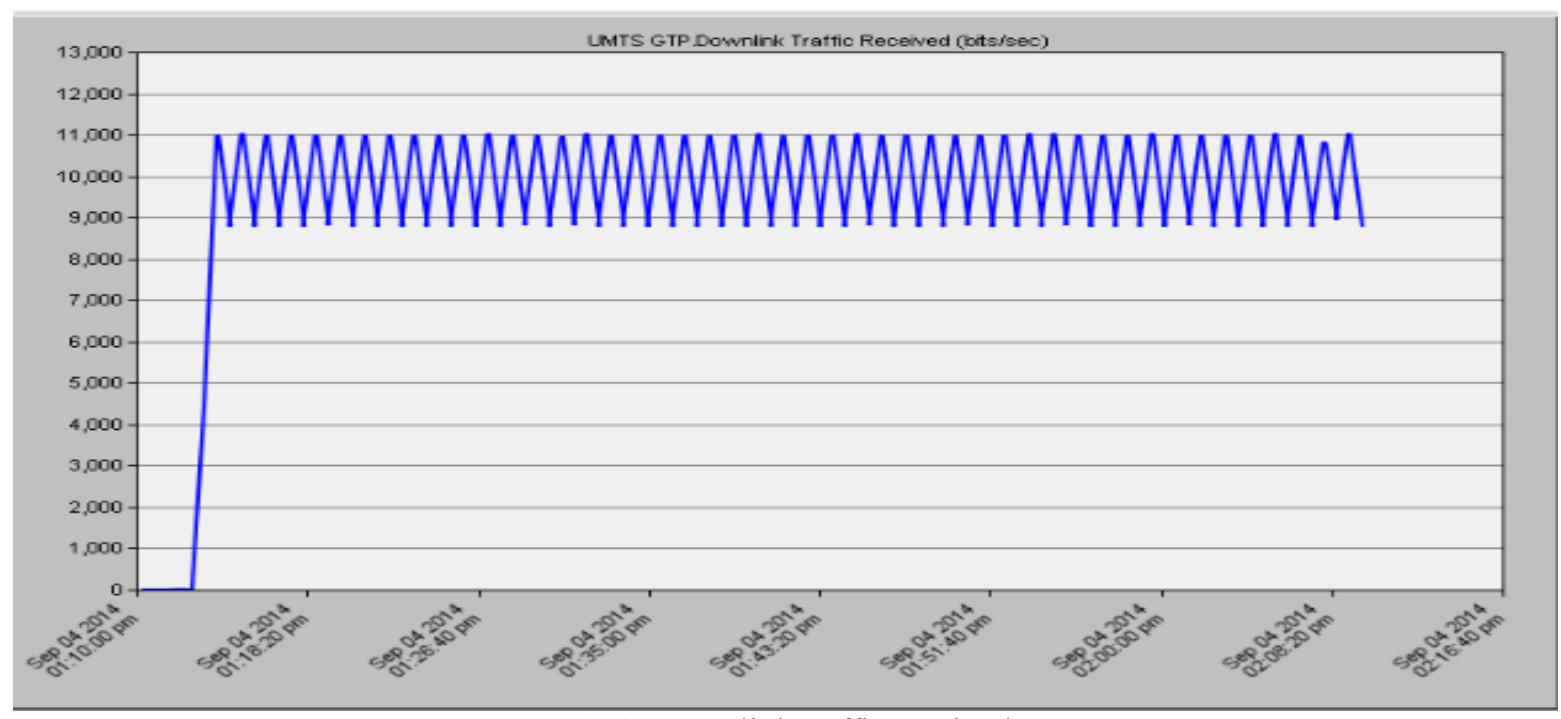

Fig 8: Downlink traffic received 


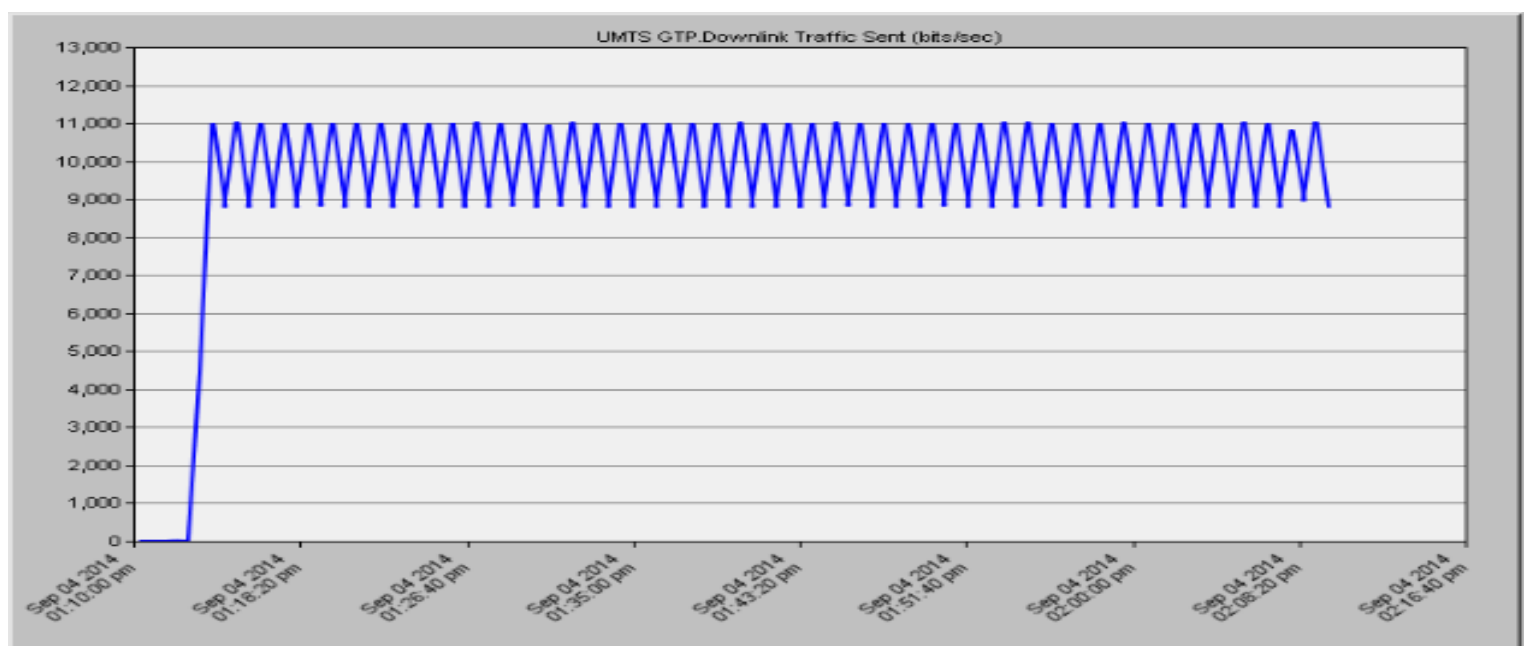

Fig 9: Downlink traffic sent

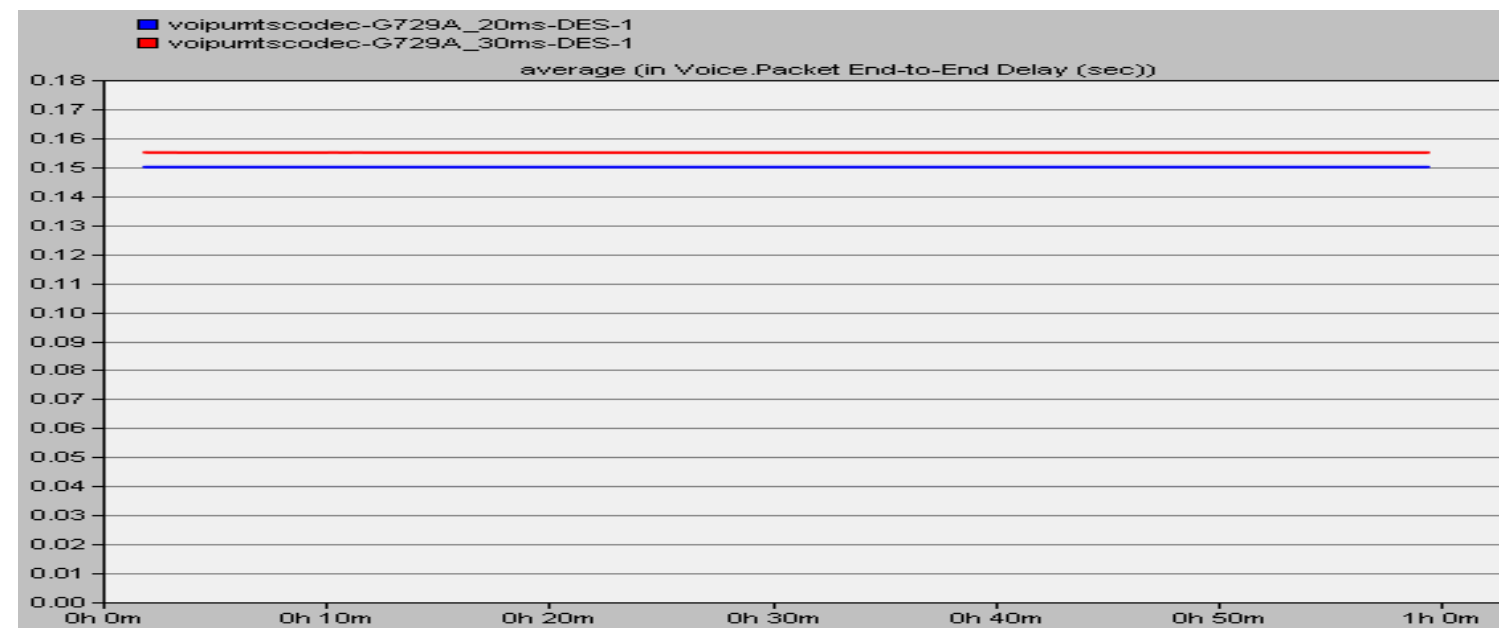

Fig 10: Average in (voice packet end to end delay) by using the G729A CODEC in frames (20, $30 \mathrm{~ms})$

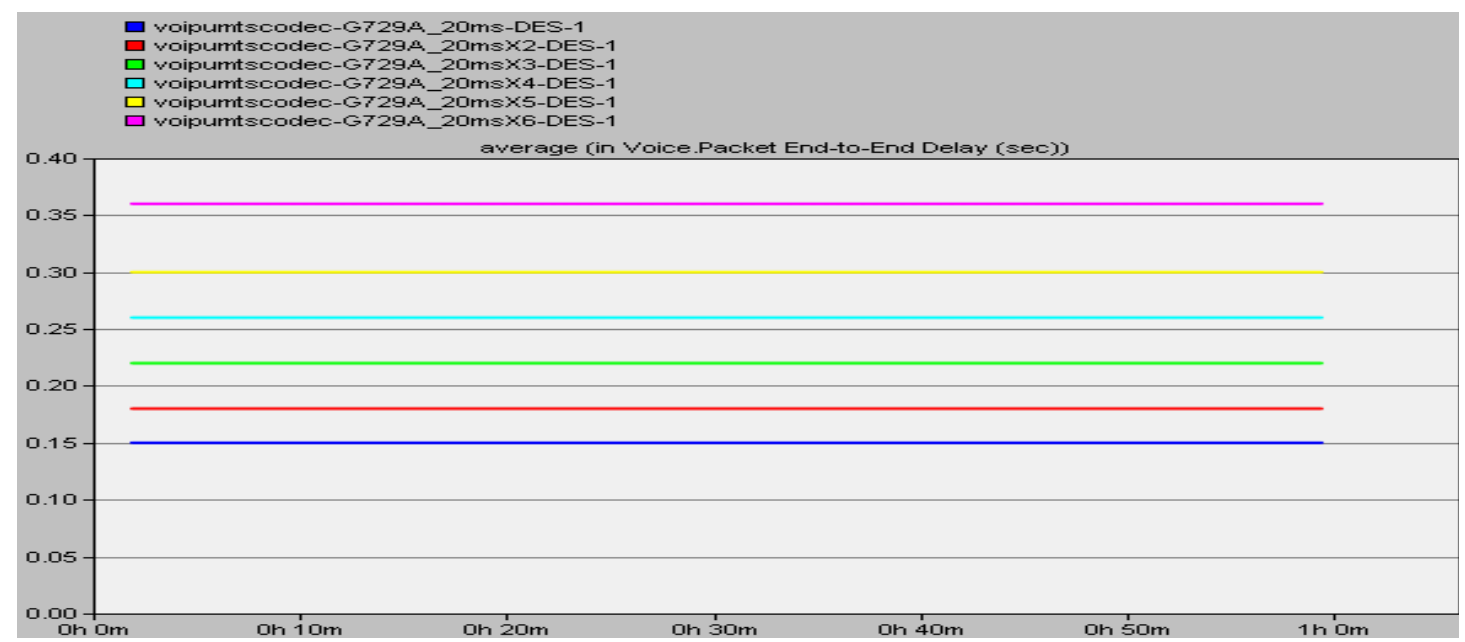

Fig 11: average in (voice end to end delay for G.729 CODEC with multiple 20ms frame size) 


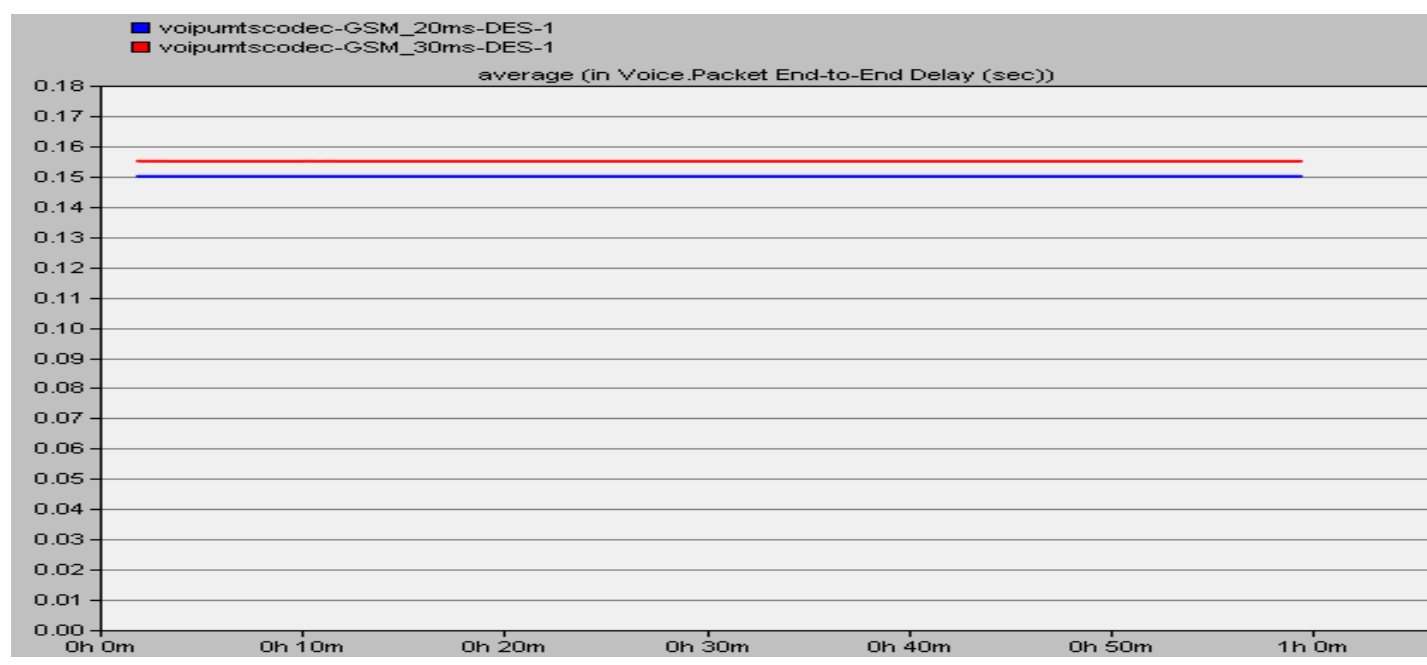

Fig 12: Average in (voice packet end to end delay) by using the GSM CODEC in frames (20, $30 \mathrm{~ms})$

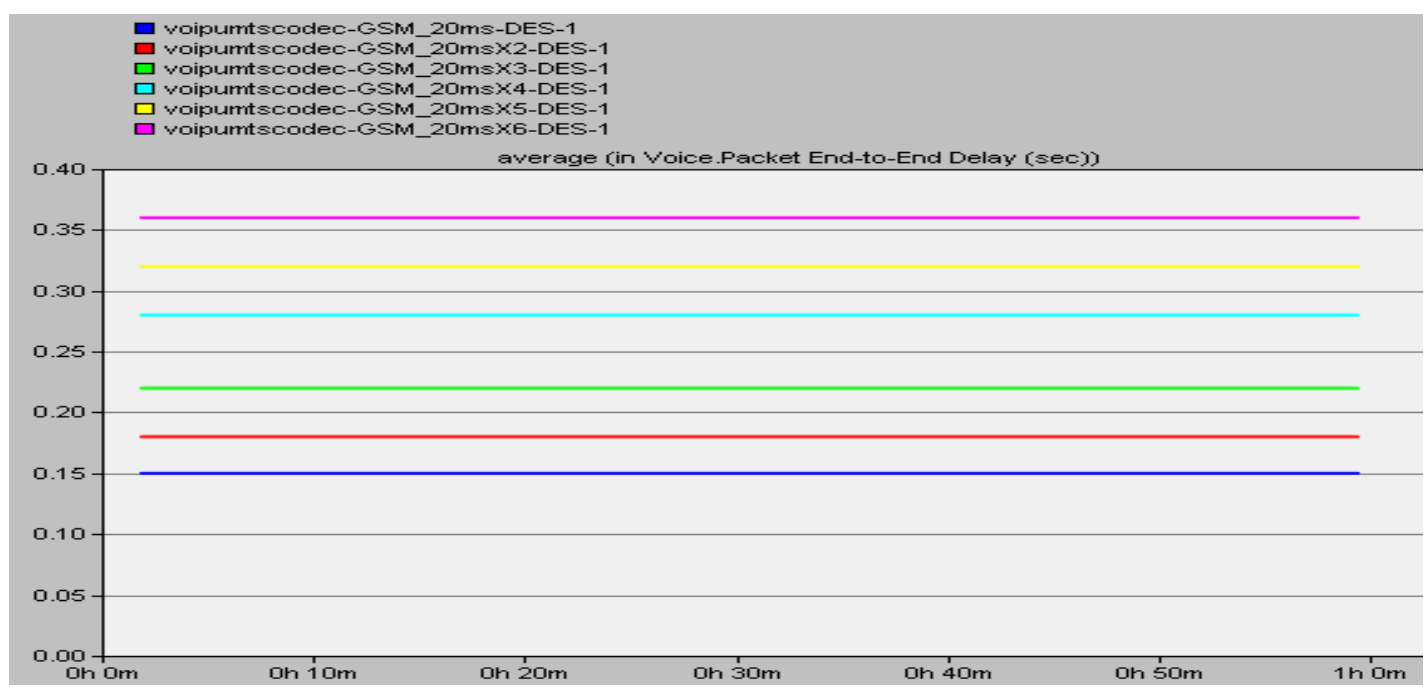

Fig 13: average in (voice end to end delay for GSM CODEC with multiple 20ms frame size)

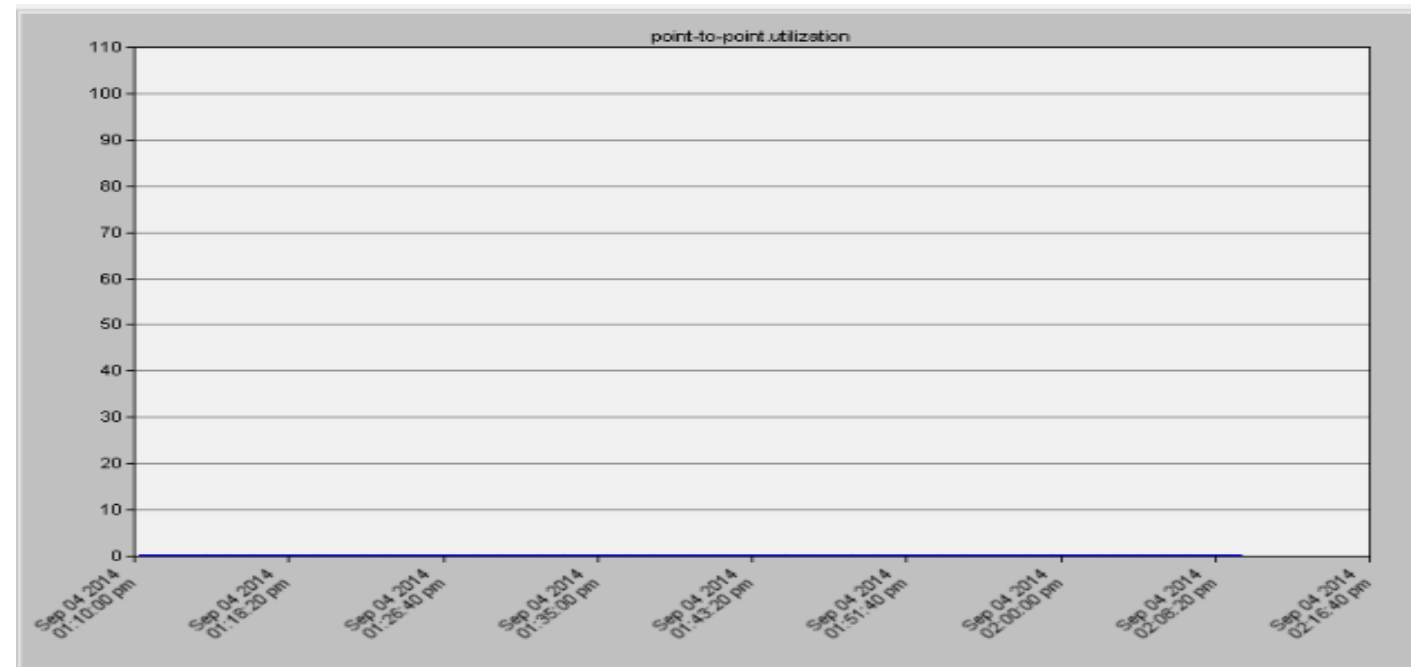

Fig 14: Utilization 
Tables:

\begin{tabular}{|c|c|c|c|c|c|c|}
\hline \multirow[t]{2}{*}{ Medium } & \multirow[t]{2}{*}{ Application } & \multirow[t]{2}{*}{$\begin{array}{l}\text { Degree of } \\
\text { symmetry }\end{array}$} & \multirow{2}{*}{$\begin{array}{l}\text { Data } \\
\text { Rate } \\
\text { (Kbps) }\end{array}$} & \multicolumn{3}{|c|}{$\begin{array}{l}\text { Key performance parameters and target } \\
\text { values }\end{array}$} \\
\hline & & & & $\begin{array}{l}\text { End-to- } \\
\text { end one } \\
\text { way delay } \\
\text { (ms) }\end{array}$ & \begin{tabular}{|l} 
Delay \\
variation \\
within a call \\
(ms)
\end{tabular} & $\begin{array}{l}\text { Information } \\
\text { loss }\end{array}$ \\
\hline Audio & $\begin{array}{l}\text { Conversational } \\
\text { voice }\end{array}$ & Two-way & $4-25$ & $\begin{array}{l}<150, \\
\text { preferred } \\
<400 \\
\text { limit. }\end{array}$ & $<1 \quad$ (jitter) & $<3 \%$ FER \\
\hline Video & Video phone & Two-way & $32-384$ & $\begin{array}{l}<150, \\
\text { preferred } \\
<400 \\
\text { limit. } \\
\text { Lip- } \\
\text { synch. } \\
<100 \mathrm{~ms}\end{array}$ & $<1$ & $<1 \%$ FER \\
\hline Data & $\begin{array}{l}\text { Telemetry two- } \\
\text { way control }\end{array}$ & Two-way & $<28.8$ & $<250$ & $\mathrm{~N} / \mathrm{A}$ & Zero \\
\hline Data & $\begin{array}{l}\text { Interactive } \\
\text { games }\end{array}$ & Two-way & 1 & $<250$ & $\mathrm{~N} / \mathrm{A}$ & Zero \\
\hline Data & Telnet & $\begin{array}{l}\text { Two-way } \\
\text { (asymmetric) }\end{array}$ & 1 & $<250$ & $\mathrm{~N} / \mathrm{A}$ & Zero \\
\hline
\end{tabular}

Table [1]: UMTS QoS requirement [1]

\begin{tabular}{|c|c|c|}
\hline CODEC & Coding Algo & Sampling rate \\
\hline GSM-FR & PRE-LTP & 13 kbps \\
\hline G.711 & PCM & 64 kbps \\
\hline G.723.1 & ACELP & $5.3 \mathrm{kbps}$ \\
\hline G.729A & CS-ACELP & 8 kbps \\
\hline
\end{tabular}

Table [2]: Characteristics of VoIP CODECS [14]

\section{Analysis}

In this section the simulation results will be presented and discussed. Simulation results are displayed. Fig 2 shows the packet loss value, Packet loss does large damage to the signal, as resending cannot be taken as an option while transferring voice. We utilized the advanced error detection and correction algorithms to fill the blank done by the fallen packets. A voice is warehoused and is utilized to do a new voice from an algorithm which endeavor to thereabout the contents of the damage packets or lost packets and according to result in fig 2 the simulation gives best result Figures [3,4,5,6,7,8and 9 ] evaluating the capability of wireless networks for VoIP transmissions, since they provide measures for the impact of the network on the perceived speech quality, the QoS factors will be controlled and managed to ensure good quality VoIP call, the design of layered coding and multiple description coding is employed to address the bandwidth fluctuations and packet loss problems in the wireless network and to further enhance the error resilience, Figure 10 and Figure 12 show GMS and G.729A CODEC get less end to end delay when they are using $20 \mathrm{~ms}$ frame sizes than $30 \mathrm{~ms}$ frame sizes. The actual end to end delay time is very close. Both CODECs are getting about $150 \mathrm{~ms}$ end to end delay for a $20 \mathrm{~ms}$ frame size and $158 \mathrm{~ms}$ end-to-end delay for a $30 \mathrm{~ms}$ frame size. The results shown in Figure 10 and Figure 12 are acceptable for VoIP calls. Figures 11 shows the results for GSM CODEC with Six simulations have using the hybrid network with a $20 \mathrm{~ms}$ frame size has been configured and used with a varied number of voice call frames in each VoIP packet. As shown in Figures 11 increasing the number of voice call frames per VoIP packet will increase the end to end delay. The $20 \mathrm{~ms}$ frame size still performs better than other length frame sizes with about $150 \mathrm{~ms}$ end to end delay and with a varied number of voices call frames in each VoIP packet about 365 as maximum value and 150 as minimum value. In Figure 13 show similar results were found for the G.729A CODEC. The simulations carried out were to identify if changes in the number of voice packets 
and CODECs affected the performance overall. Research has confirmed that packet delay of $150 \mathrm{~ms}$ doesn't do any problem, but if the delay grows up to $150 \mathrm{~ms}$ the voice signal is damage. The service providers have to guarantee that the delay happen is equal or less than $150 \mathrm{~ms}$.Fig 14 shows utilization.

\section{Conclusion}

From simulation, we observe that the voice quality of service in 3G/UMTS networks is influenced by several factors such as delay, throughput and packet loss.

Packet loss and delay are the key parameters to measure the performance of QoS voice transmission in the Network; we have observed that the packet loss and delay can occur due to several reasons. One reason is large frame size and another is network congestion. The frame size should be properly sized for proper voice transmission.

On the basis of results attained on simulation model, results showed that In UMTS network the best VoIP quality is given while using GSM voice calls with $20 \mathrm{~ms}$ frame sizes achieved better end to end delay results than voice calls with $30 \mathrm{~ms}$ frame sizes and much better results than for the other frame sizes. In this section voice calls with a $20 \mathrm{~ms}$ frame size will be used to investigate the relationship between end to end delay and voice call frames per packet, the design of layered coding and multiple description coding is employed to address the bandwidth fluctuations and packet loss problems in the wireless network and to further enhance the error resilience, Packet losses affect the quality of received voice data, We have selected these delays as metrics to measure the performance of voice quality of service. Throughput is another important parameter to measure the data rates of communication in the network. In our case, we choose throughput to measure the data rates for voice transmission in $3 \mathrm{G} / \mathrm{UMTS}$ network, overall the results showed that as more voice call frames are added to each VoIP packet the end to end delay will increase. The obtained results suggest that such an approach could enhance the native UMTS QoS.

\section{Acknowledgements}

I am very grateful to my parents Bakri Abd elhaleem Derar and Mofida Saeed Mohammed Ahmed Alfahal who have always given me their unconditional caring and support, this thesis is dedicated to them.I wish to express my deepest gratitude to my supervisor Professor Amin Babiker Abd elnabi for showing great interest in my work and for the guidance that he has given me.

\section{References}

[1]. Qinqing Zhang Mooi Choo Chuah, Design and Performance of 3G Wireless Networks and wireless LANS.: Springer Science-1Business Media, Inc., 2006.

[2]. 3GPP. Technical Speci_cation Group Services and System Aspects - Policy and charging control architecture (Release 9). TS 23.303, March 2009 .

[3]. Tasuka, "VoIP with SIP."

[4]. J. Cumming, "SIP market overview," 2003.

[5]. G. americas, "Why SIP-I: a switching core protocol recommendation for GSM/UMTS operators," 2007.

[6]. J. W. Seo, et al., "A study on the application of an AMR speech codec to VoIP," 2001.

[7]. R. Cuny and A. Lakaniemi, "VoIP in 3G networks: An end-to-end Quality of Service analysis."

[8]. J. Matta, et al., "A source and channel rate adaption algorithm for AMR in VoIP using the Emodel," pp. 92-99.

[9]. J. Cao, "An E-model implementation for QoS across a hybrid UMTS network," Masters, RMIT University, 2009.

[10]. F. Poppe, et al., "Guaranteeing quality of service to packetised voice over the UMTS air interface," presented at the IEEE, 2000.

[11]. F. Poppe, et al., "Choosing the UMTS air interface parameters, the voice packet size and the dejittering delay for a voice-over-IP call between a UMTS and PSTN party," presented at the IEEE INFOCOM, 2001.

[12]. R. C. Hampshire, et al., "Dynamic pricing to control loss systems with quality of service targets," 2008.

[13]. L. He and J. Walrand, "Pricing and revenue sharing strategies for Internet service providers."

[14]. Naveed Iqbal, Ajmal Khan, Malik Ahsan Ali, Uzma Anwar, Burhan Ullah, M. Faizan Sabir, " Performance Analysis of Soft and Hard Handovers based on UMTS QoS Traffic Classes," ICIIT, 2010.

[15]. H. Schulzrinne "RTP: A Transport Protocol for Real-Time Applications" (RFC3550), 2003

[16]. IETF Network Working Group "SIP: Session Initiation Protocol" (rfc 3261), June 2002

[17]. H. Arora and H. Sethu, "A simulation study of the impact of mobility on performance in mobile ad hoc networks," in Proceedings of the Applied Telecommunication Symposium San Diego, California, April 14-18, 2002. 